\title{
Frontières
}

\section{Des fleurs et des pierres}

\section{Mémoires et morts dans l'espace public en Pologne}

\section{Nicoletta Diasio}

Volume 19, numéro 1, automne 2006

Enjeux politiques et mort

URI : https://id.erudit.org/iderudit/016639ar

DOI : https://doi.org/10.7202/016639ar

Aller au sommaire du numéro

Éditeur(s)

Université du Québec à Montréal

ISSN

1180-3479 (imprimé)

1916-0976 (numérique)

Découvrir la revue

Citer cet article

Diasio, N. (2006). Des fleurs et des pierres : mémoires et morts dans l'espace public en Pologne. Frontières, 19(1), 69-73. https://doi.org/10.7202/016639ar

Ce document est protégé par la loi sur le droit d'auteur. L'utilisation des services d'Érudit (y compris la reproduction) est assujettie à sa politique d'utilisation que vous pouvez consulter en ligne.

https://apropos.erudit.org/fr/usagers/politique-dutilisation/
Cet article est diffusé et préservé par Érudit.

Érudit est un consortium interuniversitaire sans but lucratif composé de l’Université de Montréal, l'Université Laval et l'Université du Québec à Montréal. Il a pour mission la promotion et la valorisation de la recherche. https://www.erudit.org/fr/ 


\section{Des fleurs et des pierres MÉMOIRES ET MORTS DANS L'ESPACE PUBLIC EN POLOGNE}

\author{
Nicoletta Diasio, \\ Université de Marc-Bloch, Strasbourg.
}

Dix-sept ans sont passés depuis l'effondrement du communisme en Pologne, mais Varsovie est encore un grand chantier. Rien n'est plus comme «avant»: la toponymie, le nom du pays et son symbole, les monuments, le calendrier des fêtes nationales, le paysage urbain modifié par une construction intensive qui accorde aux banques la place d'honneur. Les transformations politiques, économiques et sociales qui ont eu lieu après 1989 s'inscrivent dans le corps de la ville et rendent concret le recouvrement public d'un monde de souvenirs jadis discrets, policés et confinés à l'espace privé. Comme dans d'autres pays du bloc soviétique, le «murmure de la mémoire» (Nora, 1997) y devient polyphonie, et parfois bruit assourdissant et cacophonique. La fin du grand récit communiste a permis l'éclosion de mémoires plurielles revendiquant une légitimité propre, affirmant des points de vue hétérogènes, quelquefois en conflit, et mobilisant de nouveaux acteurs sur la scène sociale ${ }^{1}$. La commémoration des morts dans l'espace public est un des modes de légitimation politique et sociale des «mémoires retrouvées» (Brossat et al., 1990).

Le retour de ces mémoires se manifeste d'abord timidement, au début des années 1980, avec les revendications du syndicat Solidarnosc. Après l'établissement de l'état de guerre du 13 décembre 1981, des croix de fleurs et de bougies, souvent à proximité des églises (par exemple celle de Sainte-Anne dans la vieille ville), per- mettaient aux Varsoviens de se retrouver autour de mémoriaux antitotalitaires et de signifier une résistance d'autant plus tenace qu'elle était toujours prête à se renouveler. Si les fleurs venaient à faner, les bougies à s'épuiser et les incursions de la police à détruire les témoignages de protestation et de fidélité, une nouvelle croix pouvait être recomposée pendant la nuit à quelques mètres de distance ou sur le lieu même de la précédente. Le caractère provisoire et fragile de ces lieux de mémoire soulignait, de façon paradoxale, leur possibilité de durer à travers des pratiques sociales: apporter des fleurs fraîches et les entretenir, allumer des cierges, prier, veiller, reconstruire. Croix et bouquets renvoyaient à un hommage cérémoniel aux défunts et renouvelaient la matrice sacrificielle et messianique des représentations de la Pologne en tant que "Christ des nations ", selon la formule très connue du poète national Adam Mickiewicz. La richesse sémantique des fleurs permettait de redéployer cette image sacrificielle, messianique et combattante, qui s'affirme sur la scène européenne au début du XIX ${ }^{\mathrm{e}}$ siècle, après une longue préparation qui a fait de ce pays, dès la fin du Moyen Âge, «l'avantposte de la Chrétienté ${ }^{»}$.

L'avènement de la société postcommuniste s'est manifesté, par contre, dans un délire de pierre qui a signifié à la fois la reconstruction économique et l'établissement ou la restauration de monuments faisant référence à des faits qui, dans le passé, avaient été occultés, manipulés ou réinterprétés par le discours politique officiel. 
Éphémères, les fleurs marquaient la permanence de l'opposition entre «nous» et "eux ${ }^{3}$. La pierre consent aujourd'hui d'asseoir la légitimité souvent précaire de nouveaux pouvoirs en place et de reconnaître le bien-fondé d'une société plurielle, fondée moins sur l'antagonisme que sur la reconnaissance et la recomposition. Ce qui ne va pas de soi, dans un monde malade d'incertitude: des expressions comme «ce n'est pas sûr», «on ne sait jamais», "fluctuations du marché», "insécurité », « hasard », «évènements subits », «tout peut changer d'un moment à l'autre » jalonnent les entretiens et confèrent aux souvenirs la capacité de baliser le temps et jongler avec la précarité. Comme l'histoire, les monuments «font l'objet d'une construction dont le lieu n'est pas le temps homogène et vide, mais celui plein d'actualité »(Benjamin, 1982, p. 83). Dans une ville qui, depuis des siècles, lutte inlassablement et passionnément pour restituer le passé, ces mémoriaux cristallisent des processus d'érosion karstique de la mémoire collective, qui ont commencé bien avant l'effondrement du système communiste. Ces changements ont anticipé les transformations politiques et donné lieu à un tableau mouvant, un répertoire mémoriel où se stratifient des thèmes, des personnages, des "grappes» de sens prêtes à faire surface dès qu'une nouvelle constellation sociale le demande.

\section{LE REGARD DÉTOURNÉ}

La fin $\mathrm{du}$ totalitarisme en Pologne a signifié un «bond de tigre dans le passé » (Benjamin, 1982, p. 84). Dans les années qui ont suivi 1989, les publications historiques ont été de véritables best-sellers, avec l'apparition d'une conscience historique plus nuancée et pluraliste, centrée non seulement sur la réminiscence de la double occupation nazie et soviétique, mais aussi sur la remémoration de la présence juive en Pologne, de l'expulsion des Allemands à la fin de la Deuxième Guerre mondiale et du questionnement sur le sort des minorités ethniques et religieuses avant 1939 et après 1945 . Néanmoins, d'après Ziolkowski, la rupture radicale dans l'espace et dans le temps, a produit une «mémoire particulièrement sélective. S'il y a une bonne connaissance des faits du passé, on remarque aussi des formes d'amnésie culturelle, quant à la mémoire polonaise de la présence allemande dans la Pologne contemporaine et à la mémoire que les voisins orientaux de l'Ukraine et de la Biélorussie entretiennent sur la présence polonaise et ses atouts dans leurs propres territoires» (Ziolkowski, 2000, p. 304).

L'amnésie culturelle dont parle Ziolkowski me paraît plutôt un regard détourné qui compose des images plurielles du pays. Dans les récits généalogiques recueillis dans l'espace domestique, l'évocation des marches orientales, les changements de frontières, les migrations forcées sont au cœur de la mythologie familiale et entretiennent l'idéal nostalgique d'une Pologne plurilinguistique et pluriculturelle. Le regard de loin se nourrit de la diversité et des vicissitudes qui composent le roman familial. Par contre, si on encourage les informateurs à déplacer leur regard de l'entre-soi familial à la scène nationale, les souvenirs s'exténuent dans l'évocation d'une nation à la souveraineté fragile, menacée par des étrangers internes. Ces observations rejoignent le travail de Szacka (1990) sur la transmission de la mémoire collective au sein de la famille polonaise entre 1965 et 1988 . Dans le temps, la perception d'une communauté unie par la culture, les sciences et les arts, décline pour faire place à l'image d'un État puissant et indépendant, à défendre avec les armes; une séduction pour les personnalités «fortes» vient remplacer le sentiment d'une communauté de valeurs. La transition au nouvel ordre politique postcommuniste donne la place d'honneur au maréchal Pilsudski, au détriment d'une plus grande diversité des héros nationaux dans les données recueillies en 1965 et $1977^{4}$. La réapparition des statues du maréchal, des pierres contre des fleurs, consentie par la fin du communisme, n'est donc pas impromptue, elle s'intègre dans un processus de redéfinition de l'histoire nationale et de la notion de "polonité ${ }^{5}$. Le déphasage entre l'accent porté publiquement sur l'image de la Pologne contemporaine comme nation forte et indépendante et celle transmise et racontée au sein de l'espace domestique d'une patrie de marges et de marches, répond à des logiques de légitimité différente, l'une de type politique, l'autre identitaire, les deux coïncidant rarement dans un pays qui s'est souvent affirmé en dehors, sinon contre l'État.

La liturgie des fleurs et des bougies n'a pas néanmoins disparu de l'espace cérémoniel polonais, mais se manifeste désormais dans le cadre de gestes privés ou familiaux, spécialement à l'occasion de la célébration des morts, une véritable fête qui s'étale sur plusieurs jours et ne se limite pas à la seule commémoration des défunts proches. Des observations de terrain effectuées le 2 novembre 2004, au cimetière monumental Pawazki, montrent un bricolage entre anciennes et nouvelles sollicitudes rituelles: le primat polonais ouvre une procession religieuse suivie surtout de personnes âgées et de rares familles; dans les chemins de traverse des acteurs très connus font la quête pour la restauration des lieux de la mémoire; des jeunes demandent des autographes aux célébrités; des parents invitent les enfants, même très petits, à allumer un cierge sur les tombeaux de personnages célèbres (artistes, écrivains, chanteurs, politiciens); des adolescents déposent des fleurs et des bougies en l'honneur des partisans communistes et des insurgés de 1944; des amis se promènent en touristes. Si les pierres racontent une histoire de légitimité politique, les fleurs parlent d'êtres chers, en qui le groupe se reconnaît. Depuis 1990, le compte des bougies déposées en hommage aux morts célèbres est devenu une façon pour tester les humeurs du pays et de ses sympathies (Dunin-Wasowicz, 2004) ${ }^{6}$. Mais le culte attentionné aux héros de l'histoire nationale, cette chaîne d'affects et de contacts qui lie les vivants et les morts sur les lieux publics, sont aussi décrits par une informatrice à ses enfants comme "ce qui nous différencie, nous les Polonais, de l'Occident»(Ola, institutrice, 51 ans). Ce zèle de la mémoire donne corps à un répertoire d'actions qui, dans un courant alternatif, migrent de l'espace privé à celui public selon le contexte sociopolitique: parfois témoignage de résistance, parfois plaisir festif et performatif d'être là, parmi et avec les autres.

\section{KATYN, OU LA GUERRE INFINIE}

Les grands évènements collectifs s'imbriquent aux tragédies personnelles par les souvenirs de la Deuxième Guerre mondiale. Mettre des noms sur les absences, colmater les brèches dans la généalogie, situer des villages dont on ne sait plus l'existence, est un art qui revient à ceux qui s'évertuent à transmettre la mémoire familiale. « La Pologne polonaise est en quelque sorte une absence de lieu et un lieu de l'absence, dont le plan et l'entrelacs des contours nationaux sont accentués par le flou des récits entre générations » (Eizner et Szurek, 1990, p. 41). La guerre donne souvent corps au mythe familial, sa résonance publique n'est rien qu'un battement de cœur amplifié. Le deuxième conflit mondial produit encore ses monuments aux absents, mais sa mémoire se libère aujourd'hui de l'obédience au credo communiste, qui avait fait de la lutte contre le fascisme la justification du régime et de la «fraternité » polono-soviétique. Dès la fin des années 1980 se sont multipliées les plaques de rue évoquant l'action des partisans de l'Armja Krajowa, l'armée du gouvernement polonais en exil. De même, le monument à l'Insurrection de 1944, inauguré à la fin des années 1980 par les autorités communistes, témoigne déjà de l'érosion d'une histoire officielle qui avait minimisé l'inaction volontaire des Soviétiques attendant, sur l'autre rive de la Vistule, que les nazis massacrent les insurgés et rasent la ville rue par rue, avant d'y entrer en « libérateurs » ${ }^{7}$. Mais ce qui symbolise le mieux la mémoire retrouvée, ce sont sans doute les multiples 


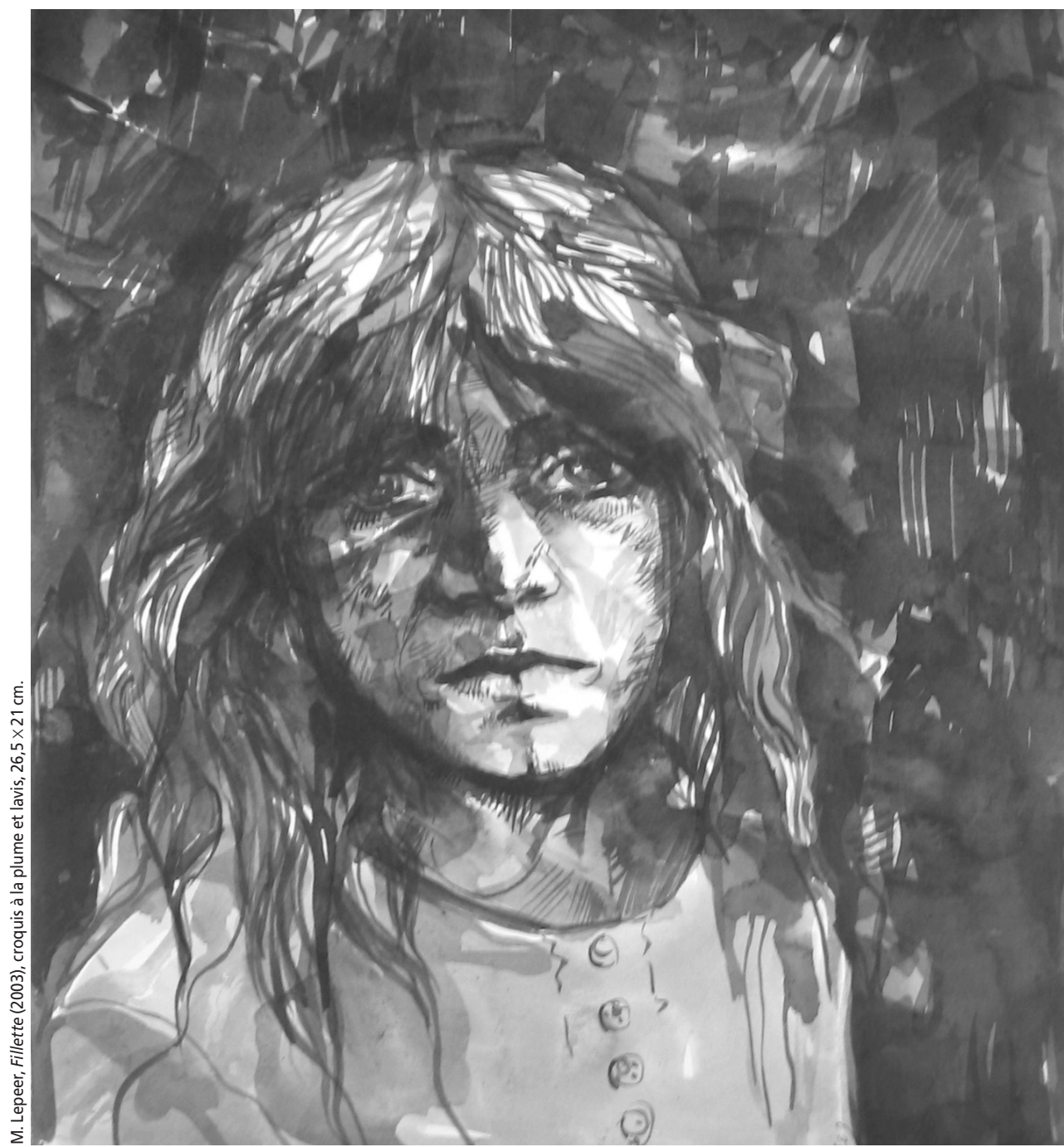

mémoriaux du massacre de Katyn, qui rappellent l'assassinat de 4500 officiers et intellectuels en mai et juin 1940 par les troupes staliniennes. À l'époque communiste, le nom même du village avait été effacé des cartes géographiques, mais le souvenir de Katyn était transmis dans «les longues conversations nocturnes des Polonais ${ }^{8} »$ et par les récits de famille palliant, par des formes de contre-histoire, les carences perçues au niveau scolaire.

Le souvenir de la Deuxième Guerre mondiale participe d'une étrange presbytie qui privilégie tout ce qui est lointain - les deux insurrections (celle du ghetto en 1943 et celle de 1944), la lutte pour l'indépendance de 1919, les luttes anti-bolcheviques des années 1920 et 1940 - au détriment des évènements de l'après-guerre. Disparues les croix de fleurs et de chandelles, aucune inscription dans la ville n'évoque l'état de guerre de 1981, un étrange silence entoure les évènements de la République populaire, aussi bien dans les récits de famille que dans les discours et les pratiques officielles. Les seuls rappels de la période communiste sont les magasins de banlieue et le culte voué au le processus de changement politique, sans entrer dans le vif des responsabilités des membres de l'ancien régime. Il est l'exemple le plus visible de ce qui me semble devenir un enkystement de la mémoire du dernier conflit mondial dans l'espace public et politique: une concrétion où se pressent les questions irrésolues de l'identité polonaise et de sa fausse évidence, et qui permet de détourner l'attention de faits plus récents et moins consensuels : des « cicatrices de la mémoire ${ }^{10}$ ». La politique de la gruba kreska ${ }^{11}$, la disparition de la rhétorique internationaliste, l'introduction de l'économie de marché n'ont pas pour autant donné lieu à un débat collectif sur les responsabilités de chacun dans le passé proche et ont sanctionné comme non punissable un système de violence, d'opportunisme et de corruption. Ce qui permet le déploiement d'un antibolchevisme confiné à des évènements lointains dans le temps et l'espace, plutôt qu'une critique aux régimes plus proches, familiers, nationaux, de Bierut, Gomulka, Gierek ou Jaruzelski, sauf à raviver, de manière stratégique, l'animosité anticommuniste pour limoger des adversaires politiques ${ }^{12}$. Elzbieta Halas parle à ce propos d'une mémoire confisquée à des fins commémoratives, d'une «amnésie collective qui rend possible la perpétuation d'un postcommunisme synthétique où la précédente eschatologie communiste de l'alliance du prolétariat et de l'intelligentsia a été remplacée par l'utopie d'une société bientôt arrivée au stade final de l'intégration européenne sous le guide d'une classe moyenne à la mission civilisatrice»(Halas, 2000, p. 319). Ces propos sont à nuancer, si on regarde la méfiance qui entoure l'Union européenne dans les discours quotidiens, mais le «synthétique» dont parle Halas, déborde du seul sens de factice. Inscrite dans le corps de la ville, aussi bien dans les petites plaques commémoratives usées par le temps que dans les grands monuments récents, la Deuxième Guerre mondiale constitue une réduction au degré zéro qui rassemble et unifie les différentes générations autour d'un temps commun: un moment de fondation de la Pologne contemporaine. Mais cette narration des origines risque aussi de perpétuer une culture du soupçon, qui continue à attribuer la responsabilité des vicissitudes polonaises aux étrangers, et de rendre évidente, donc a-historique, la configuration actuelle du pays. La centration sur la Deuxième Guerre mondiale comme moment d'élection de l'histoire polonaise réaffirme la souveraineté nationale et légitime le sacrifice économique et social au nom du retour en Europe. Les blessures du passé risquent de s'accommoder ainsi des myopies du marché. 


\section{CONCLUSION:}

\section{LE MUR QUI N'EST PAS TOMBÉ}

Ces pierres qui signifient un passé retrouvé ne remplacent pas les vestiges anciens: elles s'y superposent, dessinant un réseau de mémoires qui se croisent sans se rencontrer. Si, au début des années 1990, des auteurs ont cru à une effervescence mémorielle qui aurait amené à redessiner les contours du passé (Brossat et al. 1990), nous assistons aujourd'hui, au moins à Varsovie, à une juxtaposition, plus qu'à une combinaison, qui donne reconnaissance à des acteurs et à des histoires au pluriel, sans forcément les intégrer : l'expression de Szczypiorski à propos de la difficile cohabitation entre Polonais juifs et chrétiens - we were dying separately - semble encore d'actualité. Si, par exemple, les mémoriaux de la Shoah ont acquis une visibilité particulière par rapport à l'effacement orchestré à l'époque communiste (Szurek, 1990), les grands monuments installés par quelques fondations étrangères, comme celui aux héros du ghetto ou sur l'Umschlagplatz, lieu d'embarquement des trains pour les camps d'extermination, dessinent des espaces qui ne convergent pas avec ceux de la mémoire vivante du théâtre, de l'édition, du cimetière juif ou même du palmier qui orne l'ex-place du Parti, jadis connotée très négativement. Cette place est aujourd'hui réappropriée par la population grâce aussi à ce lien symbolique entre Varsovie et Jérusalem. Regardée à l'échelle d'une société qui «vit encore des transitions plus que de véritables transformations » (Matvejevic, 1998, p. 91), la mémoire collective ne se présente pas comme un artifice politique, établi aposteriori, mais comme un fleuve vivant qui modifie, anticipe, prépare les transformations. Ces mémoires ne sont pas uniquement libérées, mais libératrices.

Il existe enfin des pierres silencieuses, occultées, mais vivantes, vécues. Elles nous questionnent sur le sens de ces mémoriaux aux morts non seulement dans l'espace public, mais aussi dans le quotidien de ceux qui les côtoient: donnant évidence concrète aux symboles, elles façonnent des manières de s'approprier l'espace et d'en vivre les ombres et les lumières. On incorpore la mémoire, comme la marge, la mise en marge. Et à l'instar de ces basiliques romaines qui, érigées sur les lieux d'anciens temples païens, témoignent de la persistance du sacré, ces pierres sont imprégnées des souffrances d'hier et d'aujourd'hui. Ces balafres dans l'optimisme progressiste de l'histoire frappent parfois le regard extérieur, comme le montre un court extrait de mon carnet de terrain du 13 juillet 2002 :
Carnet de terrain

Varsovie - 13 juillet 2002

Le quartier où se trouvent les vestiges du ghetto se situe derrière la gare centrale, à l'ouest de l'ancien Palais de la Culture et de la Science, un immeuble imposant, aujourd'hui un souvenir, jadis un cauchemar en ciment offert par les Soviétiques, menaçant de son ombre le centre de la ville. Du parvis du Palais de la Culture, on prend la rue Zlota, au nom baudelairien "la rue dorée», encerclée par un terrain vague, un centre commercial de luxe et l'Hôtel Hilton.

On traverse une gare routière où errent, comme dans toutes les gares du monde, des désespérés. Mais la rue dorée ne mène nulle part, elle achoppe sur une espèce d'autoroute, l'avenue Jean-Paul II, qu'il faut longer pour ensuite revenir en arrière, dans un autre pré à l'abandon, élu à demeure de quelques sans-abri, pour ensuite arriver à la rue Sienna et à la continuation de la rue Zlota où restent des fragments du mur du ghetto.

Dans une cour, des tags - Kwasniewski = rabbin chef de Pologne ${ }^{13}$-, des policiers qui contrôlent les papiers à des jeunes d'une vingtaine d'années. Au numéro 64, je trouve ce fragment de mur, c'est tellement extraordinaire de pouvoir en sortir. Quelqu'un ferme une fenêtre au premier ou au deuxième étage. Une dame passe avec des sacs en plastique à carreaux bleus et rouges, jette un regard oblique.

Je retraverse la cour aux tags pour chercher les traces du mur de l'autre côté, rue Sienna. Je le retrouve au numéro 59, avec un couple de vieux très maigres, elle en jogging, lui avec un sac en plastique. D'autres personnes âgées prennent place sur un banc de pierre et bavardent, le visage tourné vers le mur. Et derrière le mur, le Palais de la Culture: les pires totalitarismes du siècle. Je pense à l'autre mur qui est tombé, en 1989, une "révolution pour les bananes", l'ont-ils appelée en Allemagne. Les gens parlent tournés vers le mur. Un ghetto dans le ghetto. D'autre nature. Mais la large voie routière et les terrains vagues n'ont pas besoin de murs et rendent vain le désir de lutter.

Ce mur fait d'espaces ouverts, de petits prés pasoliniens, de fragments d'autoroute, de vide infranchissable peut se remplir de tout fantasme, peut inventer des Juifs qui n'existent pas comme Kwasniewski et feindre que l'autre mur, celui en briques brunes, n'a jamais existé. 


\section{Bibliographie}

BENJAMIN, W. (1982[1955]) Schriften, tr. it. Angelus Novus, Torino, Einaudi.

BROSSAT, A., COMBE, S., GREENBERG, S. et al. (1990). À l'Est la mémoire retrouvée, Paris, La Découverte.

DUNIN-WASOWICZ, P. (2004). «Swiatelka o zmierzchu », Gazeta Wyborcza. Stoleczna, 2 listopada.

EIZNER, N., SZUREK J.-Ch.(1990). "Sous le regard des autres", Pologne. Dernières nouvelles de l'Atlantide, Autrement, $\mathrm{n}^{\circ} 47$, p. 41-46.

HALAS, E. (2000). "Transformation in Collective Imagination», Polish Sociological Review, vol. 3, no 131, p. 309-322.

KERSTEN, K. (1990). « De l'imagination historique », Pologne. Dernières nouvelles de l'Atlantide, Autrement, no 47, p. 19-28.

MASLOWSKI, M. (1991). "Autonomie sociale et "alliance des peuples" dans la tradition polonaise », dans M. Maslowski (dir.), L'Europe du milieu, Nancy, PUN, p. 263288.

MATVEJEVIC, P. (1998). La Méditerranée et l'Europe. Leçons au Collège de France, Paris, Stock.

MICHNIK, A. (1993). La seconda rivoluzione. L'Europa dell'Est et la costruzione della democrazia, Milan, Sperling e Kupfner.

NORA, P.(1997). Les lieux de mémoire. La République, Paris, Gallimard, tome I.

PACZKOWSKI, A. (1999). "Nazisme et communisme dans l'expérience de la mémoire polonaise », dans H. Rousso, Stalinisme et nazisme. Histoire et mémoire comparées, Bruxelles, Complexe, p. 307-330.

RAPHAËL, F. et HERBERICH-MARX, G. (dir.) (2003). Les cicatrices de la mémoire, Revue des Sciences Sociales, p. 30.

SCHRAMM, T. (2004). "Le plus grand dilemme polonais du $\mathrm{XX}^{\mathrm{e}}$ siècle: l'Insurrection de Varsovie, 1944 », dans F.-X. Cuche et J.-P. Schneider, Explorations de la mémoire collective. Grands événements et mythes des origines, Strasbourg, Presses universitaires de Strasbourg, p. 87-98.

SZACKA, B. (1990). «Społeczna pamiec polskiej przeszlosci narodowej w latach 1965-1988» dans B. Szacka et A. Sawisz, Czas przeszly i pamiec spoleczna, Warszawa, IS-UW, p. 8-120.

SZUREK, J.-Ch. (1990). "Le camp-musée d'Auschwitz » dans A. Brossat, S. Combe, S. Greenberg et al., À l'Est la mémoire retrouvée, Paris, La Découverte.

ZIOLKOWSKI, M. (2000). «Four Functions of Memory», Polish Sociological Review, vol. 3, no 131, p. 291-308.

ZAWADZKI, P. (1997). «Transition, nationalisme et antisémitisme : l'exemple polonais ", dans P. Birnbaum, Sociologie des nationalismes, Paris, PUF, p. 103-119.

ZOWCZAK, M. (1998). «Un héros de l'ombre: le père Jerzy Popieluszko », dans P. Centlivres , D. Fabre et F. Zonabend, La fabrique des héros, Paris, MSH, p. 203-210.

\section{Notes}

1. Les réflexions qui vont suivre sont le fruit de plusieurs terrains ethnographiques à Varsovie sur les modes de transmission de la mémoire familiale et les processus de naturalisation de l'appartenance. Dans ce cadre, la commémoration des morts dans l'espace public est saisie plutôt du point de vue anthropologique de la construction de sens pour les acteurs et moins dans sa genèse socio-historique telle qu'elle a pu être développée par des auteurs comme Andrzej Paczkowski (1999).

2. «La formule d'avant-poste de la Chrétienté, venue des guerres avec le monde musulman, recelait un sens important: la conscience qu'avaient les Polonais de ce que leur pays faisait office de frontière culturelle de l'Europe » (Kersten, 1990 p. 27). Comme l'explique l'historienne Kersten, cette représentation de soi fait suite à l'expansion vers l'est du pays qui, à partir de la dynastie des Piast, entraîne la Pologne dans un conflit avec la Moscovie et la Turquie.

3. La proclamation de l'état de guerre par le général Jaruzelski a lézardé cette opposition entre «nous» et "eux», qui est au cœur de l'image combattante du pays et fondée sur des multiples et tragiques expériences d'occupation: l'armée nationale qui a, par ailleurs, toujours joui d'un grand crédit en Pologne, a fait figure en décembre 1981 d'ennemi de l'intérieur.

4. Le maréchal Pilsudski a mené la lutte pour l'indépendance de la Pologne de la Russie, l'Autriche-Hongrie et l'Allemagne. Chef d'État de 1918 à 1922, il engage le pays dans une guerre contre les bolcheviks. À partir de 1926, il reprend des fonctions dans le gouvernement et instaure un régime de plus en plus dictatorial.

5. Par exemple à Varsovie, la statue du maréchal Pilsudski domine une grande place qui, jadis place de la Victoire, porte aujourd'hui son nom: au centre brille la flamme de la tombe du Soldat inconnu, encerclée d'un côté par le Jardin saxon et de l'autre par un hôtel international. Ici une grande croix de fleurs rappelait, pendant l'état de guerre, l'assassinat du père Popieluszko, commis en 1984.

6. En 2004, le personnage plébiscité, avec l'hommage de 2400 bougies, a été Jacek Kuron (1934-2004), l'un des acteurs principaux de l'opposition au régime communiste déjà dans le mouvement étudiant en 1968 et ensuite dans Solidarnosc. Ces hommages font que chaque famille, à l'approche de la fête des Morts, achète des dizaines de bougies, au supermarché, dans les épiceries, sur les étals dans la rue, dans les drogueries, les parfumeries.

7. L'histoire des passions et des controverses suscitées par l'Insurrection de Varsovie, ainsi que ses multiples lectures historiographiques entre 1950 et 1994, sont bien retracées par T. Schramm, 2004.
8. Ce verset de Mickiewicz (À une mère polonaise), écrit juste avant l'insurrection de novembre 1830 , évoque un «rite social qui a assuré en Pologne l'indépendance de l'opinion publique pendant toute la période des partages... et encore récemment, si on lit sous cet angle la formation de l'opposition polonaise» (Maslowski, 1991, p. 268).

9. Cette église est elle-même ornée d'étendards évoquant «Bóg, honor, oiczyzna» (Dieu, honneur, patrie), qui font référence à tout le martyrologe patriotique polonais et à ses dates clés. Prêtre des ouvriers de la fonderie Warszawa, le père Popieluszko a animé, par ses homélies et sa présence auprès des ouvriers en grève, la résistance anticommuniste entre 1980 et 1984. À l'âge de 37 ans, le 19 octobre 1984, il est enlevé par des agents des services de sécurité, battu et assassiné. L'image du père Popieluszko est d'ailleurs associée de façon significative à celle des victimes de Katyn ou des combattants de l'Armée de l'intérieur, emprisonnés ou assassinées dans l'après-guerre.

10. Par cette métaphore, les auteurs désignent "le souvenir non apaisé et souvent non maîtrisé d'une expérience malheureuse, [...] une mémoire meurtrie qui peut cheminer souterrainement, à l'insu de celui qu'elle taraude ou se rebiffer et affirmer avec force lorsqu'elle se heurte au soupçon, voire à la dénégation» (Raphaël et Herberich-Marx, 2003, p. 7).

11. La «ligne épaisse », c'est-à-dire le trait qui a été tiré sur le passé après 1990 et qui a clos le débat sur les responsabilités des membres de la nomenklatura du régime communiste.

12. C'est le cas des usages de la loi de «lustration », qui oblige les hauts fonctionnaires à déclarer s'ils ont ou non collaboré avec l'expolice politique communiste, par la coalition conservatrice, populiste et ultracatholique qui gouverne la Pologne en 2006.

13. A. Kwasniewski a été président de la Pologne entre 1995 et 2005. Le soupçon de "judéité » est un des moyens les plus répandus pour discréditer un politicien. Sur ce point, cf. P. Zawadzki, 1997. 